\title{
Hydrogen-bond driven intercalation of synthetic fluorinated mica by poly(oxypropylene)-amidoamine salts
}

\author{
Jiang-Jen Lin*, Yu-Min Chen, Ming-Hong Yu \\ Institute of Polymer Science and Engineering, National Taiwan University, Taipei 10617, Taiwan \\ Received 22 October 2006; received in revised form 31 January 2007; accepted 5 February 2007 \\ Available online 13 February 2007
}

\begin{abstract}
A series of organic silicate hybrids were synthesized through ion exchange reactions between the poly(oxypropylene)-amidoamine salts and the following two clays: synthetic fluorinated mica (Mica) and sodium montmorillonite (MMT). The amidoamines were prepared from the oligomerization of poly(oxypropylene)-diamine of $400 \mathrm{~g} / \mathrm{mol} M_{\mathrm{w}}$ (POP400) and dicarboxylic acid (adipic or sebacic acid) at various molar ratios of 2:1, 3:2 and 4:3. The organo-clay hybrids can achieve a high organic embedment of up to $70 \mathrm{wt} \%$, exceeding the theoretical amount (61 wt\%) as calculated from the clay cation exchange capacity $(\mathrm{CEC}=120$ mequiv. $/ 100 \mathrm{~g})$. The intercalation profile indicated a continuing increase in basal spacing from 5.8 to $7.8 \mathrm{~nm}$ for Mica and 5.2 to $7.1 \mathrm{~nm}$ for MMT when using the excess amount of POP400-sebacamide as the intercalating agent. The excess intercalation was attributed to the presence of hydrogen bonding which affect the POP-amidoamine self-aggregation within the confinement.
\end{abstract}

(C) 2007 Elsevier B.V. All rights reserved.

Keywords: Clay; Hydrogen bonding; Hydrophobic effect; Polymer/mica hybrid

\section{Introduction}

Fine dispersion of layered silicate materials in organic polymers as nanocomposites has been intensively explored [1-5]. Since the naturally abundant silicate clays are hydrophilic and incompatible with hydrophobic polymers, an organic modification is required in order to improve their affinity for polymeric materials [6,7]. The clay modification is generally performed by using organic surfactants with cationic charges such as fatty amine salts [8] and phosphonium salts $[9,10]$. The main driving force for the organics to enter the clay interlayer confinement is the cationic exchanging reaction between the organic salts and the counter ions in clays. With the organic incorporation, the silicate basal spacing is consequently expanded to the range of 2.0-3.0 nm and the resultant organoclay becomes compatible with hydrophobic polymers. However, the organoclays for polymer nanocomposites may not be suitable for the biomaterial applications. Recent research $[11,12]$ have indicated that the large biopolymers with polar groups such as phosphoric

\footnotetext{
* Corresponding author. Tel.: +88623366 5312; fax: +886233665237.

E-mail address: jianglin@ntu.edu.tw (J.-J. Lin).
}

acid, carboxylic acid, amine and their salts may be possibly intercalated into the cationic clays as well as anionic layered double hydroxides (LDH). The embedded organic species could serve as the medium for interacting with biomaterials and affect their biological functions [13]. Therefore, it is desired to tailor the organoclays with different intercalating agents, which may interact well with the versatile biomaterials.

Recently, we have developed the uses of the hydrophobic poly(oxypropylene)- and hydrophilic poly(oxyethylene)diamines of different molecular weights $(200-4000 \mathrm{~g} / \mathrm{mol})$ for intercalating with the smectic clays. The layered structures of different platelet sizes including sodium montmorillonite (MMT) and synthetic fluorinated mica (Mica) can be spatially enlarged 1.9-9.2 $\mathrm{nm}$ basal spacing [14-16]. It was also found that the extensive organic intercalation requires the formation of a hydrophobic phase in the clay gallery through the mechanism of critical mass aggregation [17]. In the intercalation profile, the layered silicates were incorporated with organics through an ionic exchange driving force, while the platelet distance was widened by a newly generated hydrophobic phase in the confinement. Hence, a suitable intercalating agent for the silicate interlayer expansion actually requires dual functionalities, amine salts for ionic exchanging and hydrophobic POP back- 
bones for spatial enlargement. The concept of POP backbone self-aggregation in clay galleries prompts us to design another class of the intercalating agents with multiple amide functionalities, besides the amine and POP backbone. After embedding into the layered silicates, the hybrids are potentially compatible with the polar biomaterials such as proteins and nucleic acids due to the potential hydrogen bonding interaction. Herein, we report the synthesis of a new class of intercalating agents and their intercalation with the clays of different dimensions.

\section{Experimental section}

\subsection{Materials}

The synthetic fluorinated mica (SOMASIF $^{\mathrm{TM}} \mathrm{ME}-100$, purchased from CO-OP Chemical Co., Japan) is a layered silicate clay with chemical composition of $\mathrm{Si}(26.5 \mathrm{wt} \%)$, $\mathrm{Mg}$ (15.6wt\%), Al (0.2wt\%), Na (4.1wt\%), Fe (0.1wt $\%)$, $\mathrm{F}(8.8 \mathrm{wt} \%)$ and cationic exchange capacity (CEC) of 120 mequiv./100 g. Sodium montmorillonite (MMT), supplied by Nanocor Co., is an $\mathrm{Na}^{+}$form of layered smectite clay with a cation exchange capacity (CEC) of 120 mequiv./100 g. Poly(oxypropylene)-diamines (POP-amine) of $M_{\mathrm{w}}=400$, 2000, and $4000 \mathrm{~g} / \mathrm{mol}$ (abbreviated as POP400, POP2000 and POP4000, accordingly) were obtained from Huntsman Chemical Co. or Aldrich Chemical Co. Sebacic acid and adipic acid were purchased from Lancaster Synthesis Ltd. and used as such without further purification.

\subsection{Synthesis of poly(oxypropylene)-amidoamines}

The preparative procedures for the POP-segmented amidoamines at various molar ratios of POP400-diamine and dicarboxylic acid $\left(-\mathrm{NH}_{2} /-\mathrm{COOH}\right.$ equivalent ratio) were reported previously [18]. The typical experimental procedures are described in the followings. To a $300 \mathrm{ml}$ of three-necked, round-bottled resin flask, equipped with a heating devise, a temperature controller, a mechanical stirrer, and a Dean-Stark trap, were charged POP400 (187 g, $468 \mathrm{mmol})$ and sebacic acid $(63.1 \mathrm{~g}, 312 \mathrm{mmol})$. With stirring, the mixture was heated to $140{ }^{\circ} \mathrm{C}$ for $4 \mathrm{~h}$. During the process, water was generated and removed under nitrogen flow through a Dan-Stark trap. The reaction progress could be monitored by taking aliquot of sample and analyzing the amine content or amide absorption by FT-IR spectrometer. With the similar procedures, a series of POP400amidoamines were prepared from the reaction of POP400 and adipic acid or sebacic acid at 2:1, 3:2 and 4:3 molar ratios (abbreviated as POP400-AA and POP400-SA). Three adipamides, derived from adipic acid-POP400, are water-soluble in contrast to their analogous POP400-sebacamides which are water insoluble. Molecular weight distribution of these amidoamines was estimated by using a gel permeation chromatography (GPC) performed on a Waters apparatus (515 HPLC pump, 717 auto sampler, 2410 refractive index detector) with a calibration against the polystyrene standard. Fourier transform infrared spectroscopy (FT-IR) was recorded on a Perkin-Elmer Spectrum One FT-IR spectrometer in the range of $400-4000 \mathrm{~cm}^{-1}$.

\subsection{Intercalation of clays with poly(oxypropylene)-amidoamine}

The intercalation was performed by first acidifying the prepared amidoamines with equivalent amounts of aqueous hydrochloric acid to generate the corresponding amine salts, and then subjected to the ionic exchanging with the water swollen Mica or $\mathrm{Na}^{+}-\mathrm{MMT}$. The typical intercalation procedures were reported elsewhere [14], and an example for the ionic exchange reaction with the amidoamines is described below. Sodium form of synthetic fluorinated mica $(5.0 \mathrm{~g}, 6.0$ mequiv. $)$ in $500 \mathrm{ml}$ of deionized water was mechanically stirred at $50-60{ }^{\circ} \mathrm{C}$ for several hours until completely swollen. POP400-SA (4.8 g, $6.0 \mathrm{mmol})$ in a separate container was treated with hydrochloric acid $(0.22 \mathrm{~g}, 6.0 \mathrm{mmol})$ and then added to the clay slurry at $50-60{ }^{\circ} \mathrm{C}$ in one portion. After a vigorous stirring for $5 \mathrm{~h}$, the slurry was cooled to ambient temperature. The resultant precipitate was filtered, washed thoroughly with deionized water several times, centrifuged, and dried in an oven at $50^{\circ} \mathrm{C}$ for $12 \mathrm{~h}$. The basal spacing was analyzed by using an X-ray powder diffractometer (Schimadzu SD-D1 using a Cu target at $35 \mathrm{kV}$, $30 \mathrm{~mA}$ ). The $n=1 \mathrm{XRD}$ diffraction was directly observed or calculated by fitting the pattern peaks according to the Bragg's equation $(n \lambda=2 d \sin \theta)$. The organic fractions of the organoclays were measured by the decomposition curve analyzed by using a thermal gravimetric analyzer (TGA, Perkin-Elmer Pyris 1) in air. The weight losses of organic components in contrast to the inorganic residues were obtained by ramping the temperature from room temperature to $800^{\circ} \mathrm{C}$ at the rate of $10^{\circ} \mathrm{C} / \mathrm{min}$.

\section{Results and discussion}

\subsection{Synthesis of POP400-amidoamines as intercalating agents}

The synthesis of amine-terminated POP400-amide (amidoamines) was carried out by the condensation of POP400diamines and diacids including adipic acid (AA) or sebacic acid (SA) at various molar ratios for $z: z-1(z=2-4)$ according to the reaction scheme (Scheme 1). These products are actually oligomeric mixtures of several POP-segmented amines consisting of hydrophilic amide (-CONH-) functionalities and different methylene $\left(-\mathrm{CH}_{2}-\right)$ alkyls from the AA or SA diacid. During the reaction, the formation of these amidoamines were monitored by measuring the absorption of FT-IR spectrum for the presence of $1646 \mathrm{~cm}^{-1}$ (amide carbonyl, $-\mathrm{C}=\mathrm{O}$ ), $1548 \mathrm{~cm}^{-1}$ $(-\mathrm{CONH}-)$ and $1107 \mathrm{~cm}^{-1}(-\mathrm{C}-\mathrm{O}-\mathrm{C}-)$ absorptions. The analysis of gel permeation chromatography indicated their average molecular weight $\left(M_{\mathrm{W}}\right), 1800 \mathrm{~g} / \mathrm{mol}$ at polydispersity of 2.35 $\left(M_{\mathrm{w}} / M_{\mathrm{n}}\right)$ for POP400-AA $(z=3)$, and $2600 \mathrm{~g} / \mathrm{mol}$ at polydispersity of 2.26 for POP400-SA $(z=3)$. For the POP400-SA at $z=2$ and $z=4$, the GPC molecular weights $\left(M_{\mathrm{w}}\right)$ were analyzed to be 1900 and $3200 \mathrm{~g} / \mathrm{mol}$, respectively. The amine titration of 2.00 , 1.18 , and $0.90 \mathrm{mmol} / \mathrm{g}$ for three SA polyamidoamines $(z=2-4)$ could be correlated to the GPC analysis and also consistent with the theoretical calculation $(1.99,1.25$, and $0.91 \mathrm{mmol} / \mathrm{g}$, 


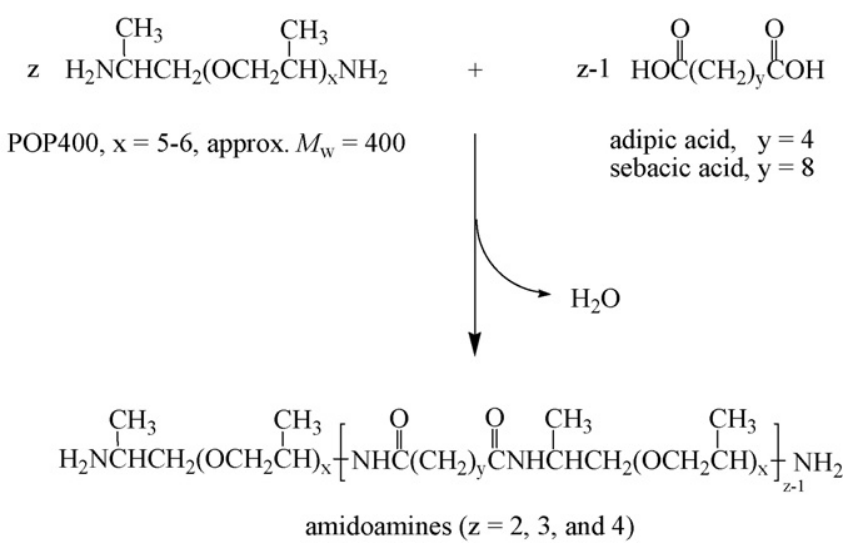

Scheme 1. Preparation of POP-amidoamines from the condensation of diamines and diacids at three different molar ratios.

respectively). The results indicated the distribution and average molecular weights in the range of $1800-3600 M_{\mathrm{W}}$ were mainly controlled by varying the molar ratios of the diamine/diacid starting materials.

\subsection{Hydrophilic and hydrophobic properties of the amidoamines}

Since both of synthetic fluorinated mica and sodium montmorillonite clays are hydrophilic and swollen in water, the intercalating amine salts are required to be soluble in water. For the starting POP-diamines, POP400 $\left(400 \mathrm{~g} / \mathrm{mol} M_{\mathrm{W}}\right)$ is watersoluble while the higher molecular weight analogs, POP2000 $(2000 \mathrm{~g} / \mathrm{mol})$ and POP4000 $(4000 \mathrm{~g} / \mathrm{mol})$, are hydrophobic and water-insoluble. However, the hydrophobic POP2000 and POP4000 amines become water-soluble when converted into the corresponding amine salts with the $\mathrm{HCl}$ addition and hence effective for clay ionic exchange reaction. The prepared POP400 amidoamines are structurally comprised of POP400 segments, amide functionalities, and hydrophobic alkyls $\left(-\mathrm{C}_{4} \mathrm{H}_{8}-\right.$ for AA and $-\mathrm{C}_{8} \mathrm{H}_{16}-\mathrm{SA}$ ). The resultant POP-adipamides (from AA) are water-soluble while the POP400-sebacamides (from SA) are water-insoluble (Table 1). It seems that the difference in alkyl lengths strongly influences the hydrophobic nature of these amidoamines. With the hydrophobic POP400-SA backbone, the corresponding amine salts are found to be effective for intercalating clays.

Table 1

Solubility and relative hydrophilicity of the starting POP-amine, POPamidoamines and their $\mathrm{HCl}$ salts

\begin{tabular}{lllll}
\hline Intercalating agent & $\mathrm{H}_{2} \mathrm{O}$ & $\mathrm{H}_{2} \mathrm{O} / \mathrm{HCl}$ & Toluene & EtOH \\
\hline POP400 & + & + & + & + \\
POP2000 & - & + & + & + \\
POP4000 & - & + & + & + \\
POP400-AA $(z=2,3$ or 4$)$ & + & + & - & + \\
POP400-SA $(z=2,3$ or 4$)$ & - & + & - & + \\
\hline
\end{tabular}

+: soluble (10 wt \% solution); - : insoluble.

\subsection{Comparative intercalation of the synthetic fluorinated mica and montmorillonite by the POP-amidoamine salts}

The montmorillonite (MMT) and synthetic fluorinated mica (Mica) have a layered silicate structure with ionic bridges in binding neighboring platelets in a primary stack. Previously, we have revealed the difference in the platelet size $(300-1000 \mathrm{~nm}$ for Mica and 80-100 nm for MMT) and intercalating results between the two clays [15]. The intercalation by using POP400, POP2000 and POP4000 afforded the hybrids of basal spacing of $1.7,3.8$ and $6.8 \mathrm{~nm}$, accordingly. By comparison, MMT was intercalated into $1.9,5.7$ and $9.2 \mathrm{~nm}$ in that order. It is noticed that the large platelet Mica is less expandable under the organic incorporation. Besides the platelet size and intercalant molecular weight, the basal spacing expansion could be affected by another factor involving the hydrophobic phase separation in the clay confinement. It is also known that the hydrophobic POP2000 backbones may self-aggregate into a secondary phase and enable to widely enlarge the silicate gallery. Generally, a maximal basal expansion was reached at equivalent exchange of the clay cationic exchange capacity or at $-\mathrm{NH}_{3}{ }^{+} / \mathrm{CEC}=1$. In other words, the intercalation or organic embedment is mainly driven by the ionic exchange reaction and the resultant XRD basal spacing is influenced by the self-aggregation of the hydrophobic POP backbones.

The series of the synthesized POP-amidoamines have the backbones of amide functionalities and also the systematical variation in molecular weights, described in the representative structures $(z=2-4)$ in Scheme 1 . The initial study on the intercalation of Mica by using POP400-AA or -SA could only reach $1.7-1.8 \mathrm{~nm}$ basal spacing at the amine/salt equivalent of $-\mathrm{NH}_{3}{ }^{+} / \mathrm{CEC}=1$, in contrast to the intercalation by POP2000 $(d$ spacing $=3.8 \mathrm{~nm})$ and POP4000 $(d$ spacing $=6.8 \mathrm{~nm})$. However, by increasing the amount of the intercalating organics to the CEC equivalent, the POP400-amidoamines continued to expand the hybrid $d$ spacing but the POP2000 was less effective for a further expansion (Table 2). For example, with an excess amount of POP2000, the basal spacing increased only slightly from 3.8 to $4.0 \mathrm{~nm}$, while the POP400-SA continued the enlargement from 5.8 to $7.8 \mathrm{~nm}$. The result was unexpected since the ionic exchange reaction was beyond the $\mathrm{CEC}$ equal equivalent.

To further illustrate the intercalation profile, the increases of $d$ spacing and organic embedment were plotted against the intercalant equivalents. As summarized in Fig. 1, three intercalation profiles of POP400-sebacamides $(z=2-4)$ in different equivalent ratios of $-\mathrm{NH}_{3}{ }^{+} / \mathrm{CEC}$ are shown. For each profile, the hybrid basal spacing is progressively expanded after the CEC ionic exchange point. In the example of POP400-SA $(z=3)$ using the amount of two-fold CEC value, a sharp increase of basal spacing from 1.8 to $5.8 \mathrm{~nm}$ and an organic incorporation from 47 to $58 \mathrm{wt} \%$ were observed. Further increases in organic embedment were observed by increasing the equivalent ratios of $-\mathrm{NH}_{3}{ }^{+} / \mathrm{CEC}$ up to 4 (Fig. 1, curve $z=3$ ). In the overall profile, the incorporation of this POP-amidoamine salt could yield an increase of basal spacing from 5.8 to $7.8 \mathrm{~nm}$, accompanied by the organic embedment from 58/42 to $70 / 30$ of organic/silicate 
Table 2

Intercalation with various equivalent ratios of $-\mathrm{NH}_{3}{ }^{+} / \mathrm{CEC}$ for MMT and Mica

\begin{tabular}{|c|c|c|c|c|c|}
\hline Intercalating agent & Equivalent ratio $^{\mathrm{a}}$ & \multicolumn{2}{|c|}{$d$ spacing by XRD $(\mathrm{nm})^{\mathrm{b}}$} & \multicolumn{2}{|c|}{ Organic fraction by TGA $(w / w)^{\mathrm{c}}$} \\
\hline None & & 1.2 & 1.2 & - & - \\
\hline \multirow[t]{2}{*}{ POP2000 } & $1 / 1$ & 5.7 & 3.8 & $63 / 37$ & $49 / 51$ \\
\hline & $2 / 1$ & 5.8 & 4.0 & $68 / 32$ & $55 / 45$ \\
\hline & $2 / 1$ & - & 1.7 & - & $30 / 70$ \\
\hline \multirow[t]{4}{*}{ POP400-SA $(z=3)$} & $1 / 1$ & 2.0 & 1.8 & $52 / 48$ & $47 / 53$ \\
\hline & $2 / 1$ & 5.2 & 5.8 & $62 / 38$ & $58 / 42$ \\
\hline & $3 / 1$ & 6.6 & 7.0 & $72 / 28$ & $65 / 35$ \\
\hline & $4 / 1$ & 7.1 & 7.8 & $73 / 27$ & $70 / 30$ \\
\hline
\end{tabular}

${ }^{\text {a }}$ Equivalent ratio: based on amine salts to the cation exchange capacity (CEC) of MMT and Mica (1.20 mequiv./g).

${ }^{\mathrm{b}} d$ spacing: measured by X-ray diffraction and calculated on the basis of the Bragg equation $(n \lambda=2 d \sin \theta)$.

c Weight fraction: organic/silicate ratio measured by TGA.

weight fraction by the TGA measurements (Fig. 1, curve TGA). For the X-ray diffraction, it is interestingly noted that the organic embedment has resulted these hybrids with a pattern of Bragg's peaks for the basal spacing of 5.8, 7.0 and $7.8 \mathrm{~nm}$ (Fig. 2), indicating the formation of a highly ordered silicate alignment. In the case of $7.8 \mathrm{~nm}$, the $n=1$ peak was calculated on the basis of Bragg's equation $n \lambda=2 d \sin \theta$ by fitting the set of three $\theta$ values. Apparently, the interlayer expansion using POP400-SA is different from that of using POP2000. In the conventional POP2000 amine intercalation, the driving force for the incorporation comes from the clay cationic exchange reaction.

For the POP400-SA $(z=4, c a .3200 \mathrm{~g} / \mathrm{mol})$ intercalation, a similar trend was observed with the increases from 2.1 to finally $8.7 \mathrm{~nm}$ (Fig. 1, curve $z=4)$. For comparison, POP400-SA $(z=2$, ca. $1900 \mathrm{~g} / \mathrm{mol}$ ) is less effective for the interlayer expansion. An increase to $3.8 \mathrm{~nm}$ at 4 equiv. CEC is shown in Fig. 1, curve $z=2$, perhaps due to the shorter backbone and less amide func-

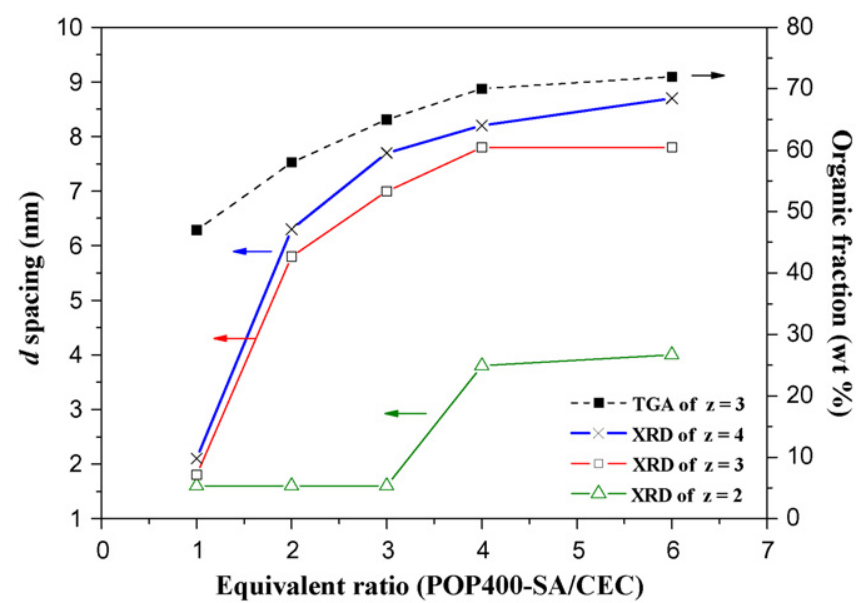

Fig. 1. Intercalation profile of POP400-sebacamides $(z=2-4)$ for Mica with respects to the changes of organic fraction and $d$ spacing in different equivalent ratios of $-\mathrm{NH}_{3}{ }^{+} / \mathrm{CEC}$. tionalities. However, all examples demonstrate the importance of the presence of amide groups that provides the additional driving force for organic incorporations beyond the CEC equivalent.

The mechanism is generalized by observing a similar trend for the MMT intercalation. As shown in Table 2, the example of POP400-SA $(z=3)$ intercalation into MMT with varied

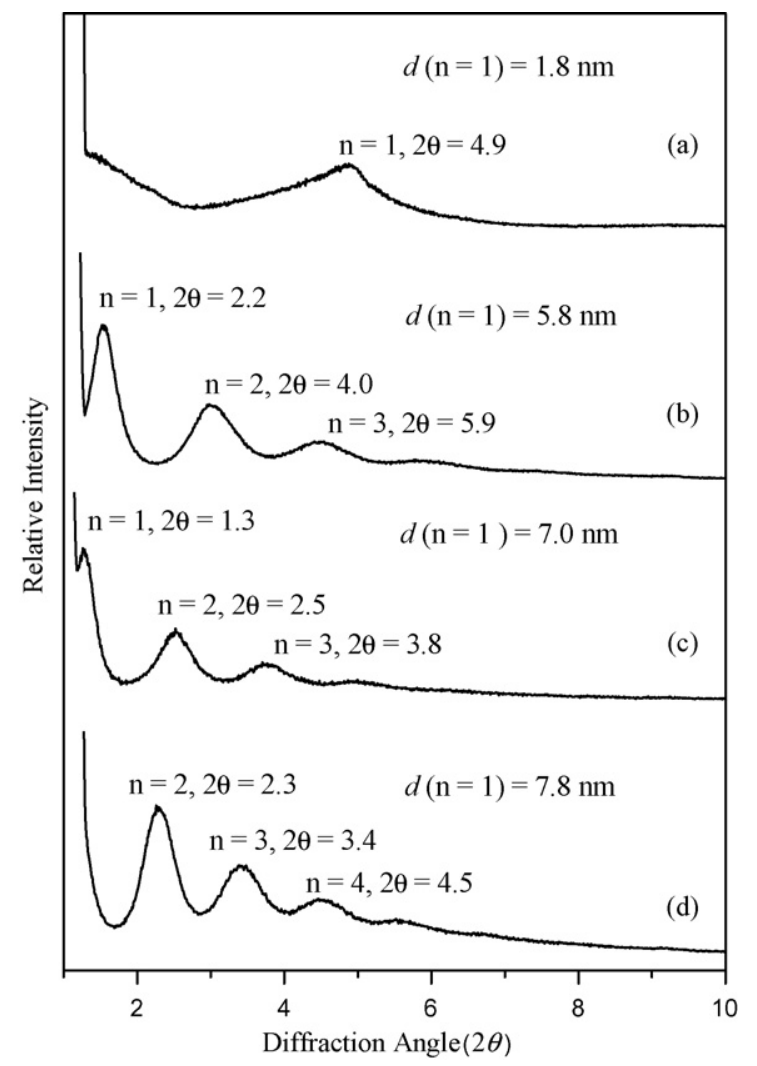

Fig. 2. X-ray diffraction patterns of Mica intercalation by POP400-SA $(z=3)$ at various $-\mathrm{NH}_{3}{ }^{+} / \mathrm{CEC}$ ratios of (a) $1 / 1$, (b) $2 / 1$, (c) $3 / 1$, and (d) $4 / 1$. 
(a)

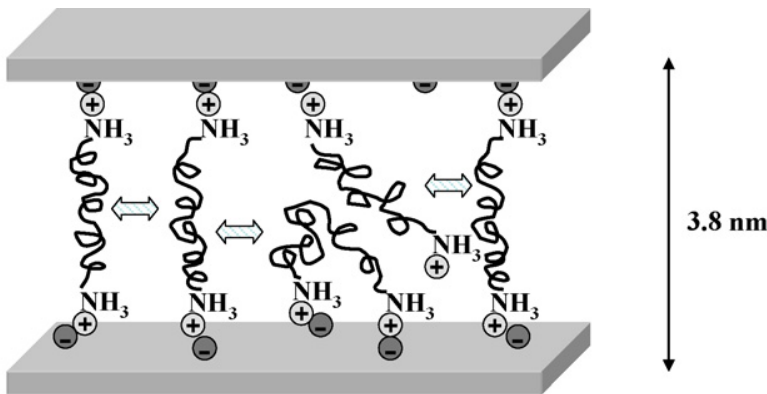

(b)

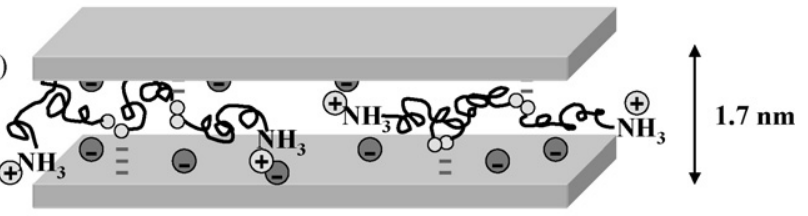

(c)

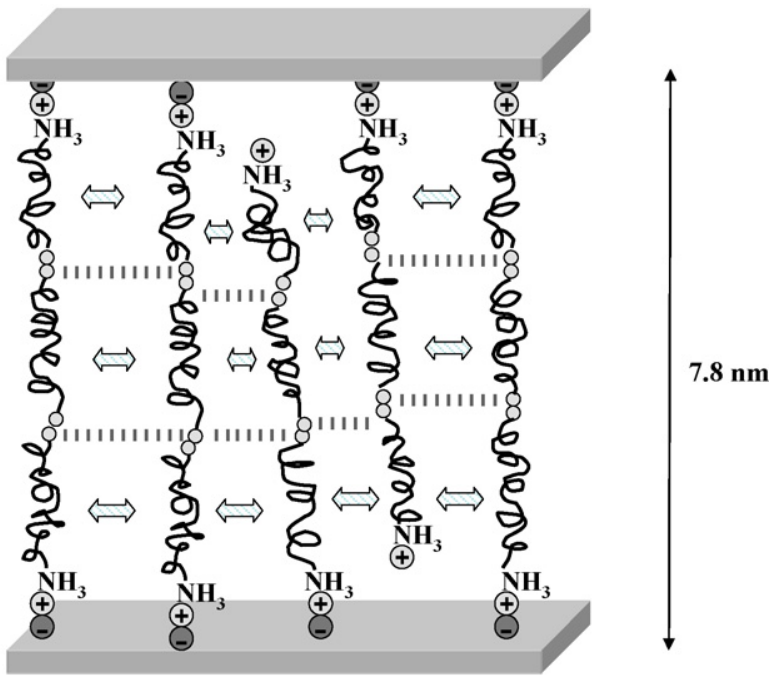

$\Leftrightarrow$ : hydrophobic effect

IIII : amide hydrogen-bonding

†/९ : ionic interaction

Fig. 3. Conceptual illustration of intercalant aggregates in the Mica gallery through ionic tethering on silicate surface, and the backbone intermolecular interactions: (a) POP2000 (POP-hydrophobic effect; XRD $=3.8 \mathrm{~nm}$ ), (b) POP400-AA (amide/ $\mathrm{Na}^{+}$interaction; XRD $=1.7 \mathrm{~nm}$ ), and (c) POP400-SA (amide H-bonding and POP hydrophobic effect; $\mathrm{XRD}=7.8 \mathrm{~nm}$ ).

$-\mathrm{NH}_{3}{ }^{+} / \mathrm{CEC}$ equivalent ratios has also achieved a progressive increase of basal spacing from 5.2 to $6.6 \mathrm{~nm}$ and then to $7.1 \mathrm{~nm}$.

\subsection{Intercalation mechanism involving intermolecular hydrogen bonding}

In the conventional intercalation, for example, the POP2000 amine-salts tend to accumulate in the Mica confinement through the POP-backbone aggregation into a hydrophobic phase [14], as illustrated in Fig. 3a. In this conceptual diagram, the POP hydrophobic phase is shown to support the platelet expansion. Such a $d$ spacing is generally large in the range of $3.8-6.8 \mathrm{~nm}$ for Mica. In contrast to the POP-amines, the POP-amidoamine salts with multiple amide functionalities in the backbone may selfaggregate differently. The poly(amidoamine)s with short alkyl
$-\left(\mathrm{CH}_{2}\right)_{4}-$ segments may incorporate and align their backbones flatly along the platelet surface through hydrophilic amide $/ \mathrm{Na}^{+}$ interaction and then exhibited a low interlayer expansion, as shown in Fig. 3b. Regardless of the amount applied, a low $1.7 \mathrm{~nm} d$ spacing and $c a .30 \mathrm{wt} \%$ of organic composition were constantly observed for the hydrophilic POP400-AA intercalation to Mica due to the lack of a hydrophobic phase. In contrast, the presence of hydrophobic $-\left(\mathrm{CH}_{2}\right)_{8}-$ alkyls in combination with amide functionalities caused the backbones to aggregate in the gallery and stretch out the neighbor platelets (Fig. 3c). It appears that both of the POP segments and amide $\mathrm{H}$-bonds contribute the formation of a hydrophobic phase in the confinement.

By comparing the FT-IR absorption of the intercalating agent and the intercalated hybrid, the presence of hydrogen bonding is possibly observable. The POP400-SA intercalating agent exhibits the characteristic absorptions of amide I and amide II $(-\mathrm{CONH})$ at 1646 and $1548 \mathrm{~cm}^{-1}$, respectively. As the hybrids were characterized with attenuated total reflection (ATR), the adsorption peaks of amide I and amide II was observed to have a shift to 1642 and $1542 \mathrm{~cm}^{-1}$. The shift of amide absorption implies the existence of hydrogen bonding interaction in the gallery [19].

\section{Conclusions}

The synthesized poly(oxypropylene)-amidoamine salts, consisting of amide hydrogen bonding in the structural backbone, enabled to intercalate into the large platelet clays different from the conventional ionic exchange mechanism. The POP400sebacamides allowed the intercalation with a profile proceeding from 1.7 to $5.8 \mathrm{~nm}$ and to $7.8 \mathrm{~nm}$ for Mica by applying excess equivalent ratios of $-\mathrm{NH}_{3}{ }^{+} / \mathrm{CEC}$. The unusual intercalation profile implies the mechanism of backbone aggregation through POP amide hydrogen bonding interaction. The new driving force for organic embedment could lead to the synthesis of new clay hybrids which are potentially compatible with biomaterials.

\section{Acknowledgment}

We acknowledge the financial support from the National Science Council (NSC) of Taiwan.

\section{References}

[1] M. Zanetti, S. Lomakin, G. Camino, Marcromol. Mater. Eng. 279 (2000) 1.

[2] B.K.G. Theng, The Chemistry of Clay-Organic Reactions, 2nd ed., John Wiley \& Sons, New York, 1974.

[3] E.P. Giannelis, Adv. Mater. 8 (1996) 29.

[4] S.S. Ray, M. Okamoto, Prog. Polym. Sci. 28 (2003) 1539.

[5] J.J. Lin, I.J. Cheng, C.C. Chu, Polym. J. 35 (2003) 441.

[6] X. Fu, S. Qutubuddin, Polymer 42 (2001) 807.

[7] H.L. Tyan, C.M. Leu, K.H. Wei, Chem. Mater. 13 (2001) 222.

[8] R.A. Vaia, R.K. Teukolsky, E.P. Giannelis, Chem. Mater. 6 (1994) 1017.

[9] W.L. Ijdo, T.J. Pinnavaia, Chem. Mater. 11 (1999) 3227.

[10] Y. Imai, S. Nishimura, E. Abe, H. Tateyama, A. Abiko, A. Yamaguchi, T. Aoyama, H. Taguchi, Chem. Mater. 14 (2002) 477.

[11] C. Causserand, Y. Kara, P. Aimar, J. Membr. Sci. 18 (2001) 165. 
[12] A. De Cristofaro, A. Violante, Appl. Clay Sci. 19 (2001) 59.

[13] J.H. Choy, S.Y. Kwak, Y.J. Jeong, J.S. Park, Angew. Chem. Int. Ed. 39 (2000) 4041.

[14] J.J. Lin, I.J. Cheng, R. Wang, R.J. Lee, Macromolecules 34 (2001) 8832.

[15] J.J. Lin, Y.M. Chen, Langmuir 20 (2004) 4261.
[16] C.C. Chou, F.S. Shieu, J.J. Lin, Macromolecules 36 (2003) 2187.

[17] J.J. Lin, I.J. Chen, C.C. Chou, Macromol. Rapid Commun. 24 (2003) 492.

[18] M.Y. Young, J.J. Lin, Ind. Eng. Chem. Res. 37 (1998) 4284.

[19] R.M. Versteegen, R.P. Sijbesma, E.W. Meijer, Macromolecules 38 (2005) 3176. 